Unfallchirurg 2003 106:707

DOI 10.1007/s00113-003-0675-9

๑) Springer-Verlag 2003

F. Kutscha-Lissberg · K.F. Hopf

\title{
Warum versagen Osteo- synthesen? - Das Kreuz mit der Biomechanik und der Biologie
}

\author{
Herrn Professor Muhr zum 60. Geburtstag
}

„Immer noch kommt es zu Implantatfehlern mit der Konsequenz von Wundheilungsstörungen oder Reoperationen. Speziell mit den neuen „biologischen“ Operationstechniken und den entsprechenden Osteosynthesematerialien treten diese Schwierigkeiten vermehrt auf. Hauptursache sind die unkritische Übernahme der traditionellen Konzepte und deren Umsetzung mit den neuartigen Techniken.

Ziel der Veranstaltung ist es, die wesentlichen Diskrepanzen zwischen bisherigen und neuen Implantaten aufzuzeigen, die Vor-aber auch Nachteile der Operationstechnik zu diskutieren und Fehlervermeidungsprogramme vorzustellen."

Mit diesen Zeilen stellte Professor Muhr das Programm des 16. Bochum-Treffs Anfang 2003 vor.

Unfallchirurgisches Handeln war im Sinne der Ergebnisqualität immer erfolgsorientiert. Durch neue Entgeltregelungen ist die Fehlervermeidung nicht nur patientenrelevant, sondern auch eine existentielle Notwendigkeit für die medizinische Behandlungseinheit. Vor dem drohenden Hintergrund der wirtschaftlich begründeten medizinischen Einschränkung bis hin zur Abteilungsschließung müssen immer größere „Fallzahlen“ kostenneutral bewältigt werden. Komplikationen, die in dieser Situation entstehen, verringern nicht nur die Behandlungsqualität, son- dern überfordern auch die strukturellen und finanziellen Ressourcen. In diesem Sinne erscheint es nicht nur sinnvoll, sondern zwingend notwendig, den perpetuierten implantat-und behandlungsassoziierten Erfolgsmeldungen die Komplikationsanalyse mehr als nur gleichwertig gegenüberzustellen.

\section{( Den Erfolgsmeldungen hinsicht- \\ lich neuer Implantate muss die Komplikationsanalyse gleichwertig gegenüber gestellt werden}

Ein wesentlicher Bestandteil chirugischen Handelns ist das permanente Hinterfragen allgemeingültiger und neuer Errungenschaften. Nur die tägliche kritische Analyse, fern vom kameradschaftlich fraternisierenden kollektiven "Schutzwall,“ ermöglicht die adäquate und rechtzeitige Reaktion im Sinne des Kompliaktionsmanagements („Ein Tag ohne Tadel ist Lob genug"). Allerdings ist die alleinige Flucht in „Neues“, und sei es nur zum Zwecke, das "Alte" nicht verstehen zu müssen, als wenig gesteuerter Aktivismus nicht geeignet, dieser Situation gerecht zu werden.

In Kenntnis der langen Tradition qualitätssichernder Maßnahmen, beginnend bei Lorenz Böhler 1917 und weitergeführt von der Arbeitsgemeinschaft für Osteosynthesefragen, können die unfallchirurgischen Gesellschaften mit Recht darauf vertrauen, dass auch diese neuen Hürden des medizinische Alltags bei konzertantem Vorgehen bewältigt werden können.

\section{F. Kutscha-Lissberg}

K.F. Hopf

\section{Korrespondierender Autor Dr. Friedrich Kutscha-Lissberg}

Berufsgenossenschaftliche Kliniken des „Bergmannsheil“ Bochum Universitätsklinik für Chirurgie Bürkle-De-La-Camp Platz 1 44798 Bochum E-Mail:Friedrich.Kutscha@bergmannsheil.de 